López-Cassá, E.; Pérez-Escoda, N. y Alegre, A. (2018). Competencia emocional, satisfacción en contextos específicos y satisfacción con la vida en la adolescencia. Revista de Investigación Educativa, 36(1), 57-73.

DOI: http://dx.doi.org/10.6018/rie.36.1.273131

\title{
Competencia emocional, satisfacción en contextos específicos y satisfacción con la vida en la adolescencia
}

\section{Emotional competence, satisfaction in specific contexts and satisfaction with life in adolescence}

\begin{abstract}
Elia López-Cassá*, Nuria Pérez-Escoda* y Alberto Alegre ${ }^{* * *}$
* Profesora asociada del Departamento de Didáctica y Organización Educativa, Universidad de Barcelona, España. ** Profesora titular del Dpto. de Métodos de Investigación y Diagnóstico en Educación, Universidad de Barcelona, España. *** Profesor adjunto del Department of Early Childhood and Elementary Education, East Stroudsburg, Pennsylvania.
\end{abstract}

\begin{abstract}
Resumen
El presente estudio analiza la relación entre el nivel de competencia emocional y el bienestar derivado de experiencias en contextos específicos así como el nivel de satisfacción con la vida en adolescentes. Se comprueban tres hipótesis: a) si la competencia emocional se relaciona con la satisfacción con la vida; b) si existe relación entre la satisfacción con la vida y con la satisfacción en diversos dominios específicos (con los estudios, con uno mismo, con los recursos de afrontamiento y con el uso del tiempo de ocio); c) Finalmente, si la relación entre la satisfacción en contextos específicos y la satisfacción con la vida es moderada por la competencia emocional. Para ello se utilizó el Cuestionario de Desarrollo Emocional para Educación Secundaria (CDE-SEC) de Pérez-Escoda, Álvarez E Bisquerra (2008), el cuestionario de satisfacción con la vida (SWLS) de Diener, Emmons, Larsen $\mathcal{E}$ Griffin (1985); adaptado al español por Atienza, Pons, Balaguer $\mathcal{E}$ García-Mérita (2000) y un cuestionario ad-hoc para medir las variables de bienestar en contextos específicos. La muestra de estudio es de 485 estudiantes de la Educación Secundaria Obligatoria de Cataluña y Bilbao. Se ha realizado un análisis de regresión mediante el que se ha obtenido un modelo explicativo de la competencia emocional y la satisfacción con la vida en los jóvenes. Los resultados confirman mayoritariamente las tres hipótesis aunque con algunos matices.

Palabras clave: competencia emocional; satisfacción con la vida; bienestar; adolescencia.
\end{abstract}

Correspondencia: Elia López-Cassá, elialopez@ub.edu. Dpto. DOE de la Universidad de Barcelona. Edificio Levante, Paseo de Vall de Hebrón, 171. 08035 Barcelona, España. 


\begin{abstract}
This study analyzes the relationship between the level of emotional competence and well-being experienced in specific contexts as well as satisfaction with life in adolescents. Three hypotheses were tested: a) If emotional competence of adolescents is related to their life satisfaction; $b$ ) If there is a relationship between life satisfaction and satisfaction in various specific domains (with studies, with oneself, coping resources and the use of leisure time). c) Finally, if the relationship between satisfaction in specific contexts and satisfaction with life is moderated by emotional competence. The Emotional Adolescent Development questionnaire (CDE-SEC; Perez-Escoda, Alvarez E Bisquerra, 2008), the Life Satisfaction questionnaire (SWLS; Diener, Emmons, Larsen $\mathcal{E}$ Griffin, 1985) adapted to Spanish by Atienza, Pons, Balaguer and García-Merita (2000) and an ad-hoc questionnaire to measure domain-specific satisfactions were used. The study sample is 485 students of Secondary Education of Catalonia and Bilbao. We performed a regression analysis by which an explanatory model of emotional competence and life satisfaction among young people is obtained. The results largely confirm the three hypotheses although with some nuances.
\end{abstract}

Keywords: emotional competence; satisfaction with life; well-being, adolescent.

\title{
Introducción
}

La adolescencia es una etapa de vida en la que surgen cambios significativos a nivel biológico, psicológico y social. Aunque no hay un acuerdo unánime sobre el concepto de adolescencia, según Moreno (2009) la adolescencia es el período de transición entre la infancia y la adultez que transcurre, aproximadamente, entre los once y doce años y los dieciocho y veinte años de edad. Scheer, Unger \& Brown (1994) definen la adolescencia, poniendo el acento en factores psicológicos, tales como la responsabilidad sobre sus acciones o la toma de decisiones autónoma.

Se trata de la etapa en la que los jóvenes se enfrentan a la difícil tarea de formar su personalidad e identidad y establecer su red relacional (Moreno, 2009; RebolloCatalán, Ruiz-Pinto \& García-Pérez, 2017). En ella, la vida emocional es muy inestable, principalmente, en los entornos, donde los adolescentes tienen más problemas: en la escuela y en casa (Schuerger, 2003). Los adolescentes se sienten incomprendidos e inseguros, acostumbran a incumplir las normas y no se esfuerzan en el orden ni en la participación (Moreno \& del Barrio, 2005). Los estudios dejan de ser importantes, no tanto por su capacidad intelectual, sino por lo que supone planificar y organizar sus tareas (Moreno, 2009). En este período, se reservan sus problemas, les cuesta mostrar sus emociones, se encierran en sí mismos y resulta difícil adaptarse a las nuevas responsabilidades sociales (López, Etxebarría, Fuentes \& Ortiz, 1999; Moreno \& del Barrio, 2005). Los estudios aportados por Rutter \& Rutter (1992) manifiestan que la ansiedad y la depresión son los problemas más característicos de la adolescencia.

Según la Convención de Naciones Unidas (1990), los adolescentes tienen el derecho de ser atendidos ante sus necesidades, pues son el futuro de la sociedad, continúan siendo personas en desarrollo y necesitadas de formación en diferentes aspectos personales y emocionales (Moreno, 2009). 
Tal como afirman Pérez-Escoda \& Pellicer (2009), Bisquerra (2011) y Proctor et al. (2015), la adolescencia es un momento clave para el crecimiento emocional. Estos autores manifiestan la necesidad de desarrollar las competencias emocionales en tanto que fortalezas para el bienestar y para el afrontamiento de diferentes situaciones en la vida de los jóvenes.

\section{Las competencias emocionales y sus dimensiones}

El concepto de Inteligencia emocional es entendido como la habilidad de las personas para atender y percibir los sentimientos, asimilarlos y comprenderlos de forma apropiada y adecuada y su destreza para regular el propio estado de ánimo o el de los demás (Salovey \& Mayer, 1990). Este constructo aporta una visión hacia el estudio de las emociones y su impacto en nuestra sociedad. El desarrollo de las competencias emocionales es el camino para la mejora de la inteligencia emocional.

Entre los científicos uno de los debates más polémicos se centra en determinar si la inteligencia emocional debe considerarse un rasgo o una capacidad. En las últimas décadas se han elaborado más de diez modelos teóricos de Inteligencia emocional (Pérez-González, 2011) aunque la mayoría de ellos más que confrontarse son bastante complementarios. Sin embargo en educación, de acuerdo con McClelland (1973) citado por Bisquerra, Pérez-González \& García-Navarro (2015) el concepto de "inteligencia" pierde su interés frente al de "competencia" ya que el primero se refiere a una cualidad o característica psicológica relativamente estable, mientras que la competencia emocional es susceptible de ser desarrollada. Es en este sentido en el cual se enmarca este estudio.

La competencia emocional se entiende como la capacidad para movilizar, adecuadamente, un conjunto de conocimientos, capacidades, habilidades y actitudes necesarias, para realizar actividades diversas con un cierto nivel de calidad y eficacia (Bisquerra \& Pérez, 2007). De acuerdo con el modelo teórico de estos autores, las competencias emocionales se estructuran en cinco dimensiones: conciencia emocional, regulación emocional, autonomía emocional, competencia social y competencias para la vida y el bienestar.

Se entiende la conciencia emocional como la capacidad para tomar conciencia de las propias emociones y de las emociones de los demás, así como la habilidad para captar el clima emocional de un contexto determinado. La regulación emocional es la capacidad para manejar las emociones de forma apropiada, en ella se incluye el dominio de estrategias de afrontamiento. La autonomía emocional se puede definir como un conjunto de características y elementos relacionados con la autogestión emocional. La competencia social se refiere a la capacidad para mantener buenas relaciones sociales con otras personas en la que se contemplan las habilidades sociales básicas y comunicación efectiva. La última dimensión emocional, las competencias para la vida y el bienestar consisten en la capacidad para adoptar comportamientos apropiados y responsables para los desafíos diarios de la vida.

Se dispone de pocos estudios sobre la influencia de la competencia emocional en la vida de los adolescentes. Algunos de ellos muestran la importancia de la competencia emocional en la autoestima, felicidad, bienestar psicológico, salud mental y satisfacción vital en la adolescencia (Furr \& Funder, 1998; Huebner, 1991a, 1991b; Lewinsohn, Red- 
ner \& Seeley, 1991; Martínez-Antón, Buelga \& Cava, 2007; Montes-Berges \& AugustoLanda, 2014; Pacheco \& Fernández-Berrocal, 2004; Ying \& Fang-Biao, 2005). También en la investigación de García-Aguilar, Viñals \& Pérez-Escoda, (2016) se evidencia que un buen nivel de competencias emocionales se relaciona con menos dificultades cotidianas en la vida de los adolescentes.

\section{La satisfacción con la vida}

Se entiende la satisfacción con la vida como una valoración global que la persona hace sobre su vida, poniendo en comparación lo que ha conseguido, con el logro de sus retos y expectativas (Diener et al. 1985; Veenhoven, 1994). Es una dimensión cuyo estudio tiene un largo recorrido, aunque adquirió enorme relevancia a partir de los años setenta, momento en el que este constructo tomó una dimensión psicosocial y humanista. Así pues, se relaciona con un sentimiento de bienestar o de felicidad y es uno de los indicadores de la calidad de vida (Pérez-Escoda, 2013; Veenhoven, 1994).

La satisfacción con la vida es un constructo que puede modificarse por elección del individuo o por variabilidad del medio ambiente y que no viene marcada totalmente por la herencia (Roysamb, Nes \& Vitterso, 2014; Tay \& Kuykendall, 2013).

Diferentes investigaciones muestran que la satisfacción con la vida está vinculada con la autoestima, el ajuste escolar, los perfiles motivacionales, los recursos de apoyo y el clima social (Cava \& Musitu, 1999; Ferragut \& Fierro, 2012; Furr \& Funder, 1998; Huebner, 1991a, 1991b; Lewinsohn et al., 1991; Martínez-Antón et al., 2007; McCullough, Huebner \& Laughlin, 2000; Moreno-Murcia \& Silveira, 2015; Pacheco \& FernándezBerrocal, 2004; Van Aken \& Asendorpf, 1997; Ying \& Fang-Biao, 2005).

\section{La satisfacción en dominios específicos}

Se entiende por dominios específicos aquellos ámbitos de la vida que se quieren evaluar en términos focales y no tan amplios. En este caso, centraremos el estudio en la satisfacción que tienen los jóvenes con los estudios, con uno mismo, con los recursos de afrontamiento y con el uso del tiempo del ocio.

Según las investigaciones de Demir \& Weitekamp (2007), Diener, Suh, Lucas \& Smith (1999), Javaloy et al. (2007) y Lyubomirsky, King \& Diener (2005) sobre los jóvenes y su nivel de satisfacción, los adolescentes muestran más satisfacción con su familia, amigos, salud y atractivo físico que con la situación económica, el ocio o los estudios. Curiosamente, aunque el tiempo libre y el estudio se consideran áreas importantes, son las que menos satisfacción produce a los adolescentes. Otras investigaciones apuntan que la satisfacción con el ocio (Kuykendall, Tay \& Ng, 2015) y la satisfacción con las relaciones (Cho \& Tay, 2015) se sitúan en un primer plano.

\section{La satisfacción con la vida y la satisfacción en dominios específicos}

La relación entre la satisfacción con la vida y la satisfacción en contextos específicos se ve apoyada por los resultados de diferentes estudios. Por ejemplo, Galíndez \& Casas (2010), con una muestra de estudiantes de secundaria demostraron que existen corre- 
laciones muy altas entre satisfacción con la vida y el autoconcepto y la autoestima. Así pues, coinciden con los trabajos de Furr \& Funder, (1998); Huebner, (1991a, 1991b) y Lewinsohn et al. (1991) al poner de manifiesto que una elevada autoestima se relaciona con una mayor satisfacción con la vida del adolescente, mientras que, por el contrario, una menor autoestima, se relaciona con una valoración negativa de la propia vida.

Otros estudios relacionan afrontamiento y bienestar en la adolescencia (GonzálesBarrón; Montoya, Casullo \& Bernabéu, 2002; Mullis \& Chapman, 2000) y concluyen que las preocupaciones de los jóvenes se centran en problemas educativos, afectivos, personales y familiares. De ellos, se concluye que las variables de afrontamiento y bienestar están estrechamente relacionadas, aunque no se puede señalar qué variable influye sobre la otra. También se ha observado que los jóvenes utilizan diferentes estrategias según el nivel de bienestar (Gonzáles-Barrón et al., 2002).

\section{La satisfacción con la vida y las competencias emocionales en adolescentes}

Entre los autores que han relacionado la satisfacción con la vida y la inteligencia emocional destacan Bermúdez, Álvarez \& Sánchez, 2003; Cazalla-Luna \& Molero, 2016; Extremera, Durán \& Rey, 2005; Ferragut \& Fierro, 2012; Páez, Fernández, Campos, Zubieta \& Casullo, 2006 y Pérez-Escoda \& Alegre, 2014. En sus estudios se destaca la relación entre el nivel de satisfacción con la vida y la dimensión emocional de la persona. Sin embargo, tan sólo son los que ofrecen Bermúdez et al. (2003) y Páez et al. (2006) están centrados en la etapa de la adolescencia.

A partir de estos antecedentes, se considera importante estudiar la relación entre el nivel de competencia emocional y la satisfacción con la vida de los adolescentes. Existe la posibilidad que determinados aspectos personales puedan influir en las experiencias vividas en determinados contextos y ello influya en el nivel de satisfacción con la vida (Ciarrochi, Chan \& Caputi, 2000; Pérez-Escoda \& Alegre (2014), en su estudio realizado con adultos comprobaron que el efecto de la satisfacción con uno mismo y con el entorno social influye en la satisfacción con la vida, y que ésta es mayor, cuando el sujeto goza de mayor competencia emocional.

Se necesitan más investigaciones, concretamente en el caso de los adolescentes, para conocer la influencia de la competencia emocional en la satisfacción con la vida en general y en algunos de sus diferentes ámbitos (estudios, conocimiento de uno mismo, recursos de afrontamiento y tiempo libre).

\section{Método}

\section{Objetivos}

Los objetivos de esta investigación son los siguientes:

1. Verificar que la relación entre competencia emocional y satisfacción con la vida que se ha observado en muestras con adultos se da también con adolescentes.

2. Comprobar que la satisfacción con la vida obedece, al menos en parte, a satisfacciones experimentadas en contextos específicos de la vida de los adolescentes. 
3. Investigar si el impacto de las satisfacciones experimentadas en contextos específicos en la satisfacción con la vida depende, al menos en parte, de la competencia emocional del adolescente.

\section{Hipótesis}

Las hipótesis de nuestro estudio son las siguientes:

Hipótesis 1: La competencia emocional predice la satisfacción con la vida de los adolescentes.

Hipótesis 2: Existe relación entre la satisfacción con la vida y algunos de los dominios específicos en los jóvenes: satisfacciones con los estudios, con uno mismo, con los recursos de afrontamiento y con el uso del tiempo del ocio.

Hipótesis 3: La competencia emocional modera la relación de predicción entre la satisfacción experimentada en cada uno de los dominios específicos y la satisfacción con la vida.

\section{Participantes}

La población estudiada es la de los adolescentes españoles. La muestra es de conveniencia y fue recogida en tres escuelas de educación secundaria, dos de Barcelona y una de Bilbao. Está compuesta de 485 estudiantes, el 55.5\% de género femenino. La edad oscila entre los 11 y los 19 años, con una media de 13.42 años y una desviación típica de 1.54. Los participantes se aplicaron los cuestionarios de forma totalmente voluntaria.

Los sujetos respondieron los cuestionarios durante el horario escolar, en clase, y con el profesor ausente. Todos los cuestionarios han sido tratados, garantizando el anonimato y la confidencialidad de los participantes.

\section{Instrumentos}

Satisfacción con la vida. Esta variable fue medida con el cuestionario "Satisfaction with Life Scale" (SWLS: Diener et al., 1985). El SWLS es, probablemente, la medida más citada de satisfacción con la vida en la literatura científica (Vázquez, Duque \& Hervás, 2012). Se compone de cinco ítems, a los que se responde en una escala tipo Likert de siete puntos, desde 1 (total desacuerdo) a 7 (totalmente de acuerdo). Diener \& González (2011) y Pavot \& Diener (2008) resumieron en dos estudios las investigaciones desarrolladas durante los últimos 20 años, que confirman las propiedades psicométricas de este instrumento. La fiabilidad de acuerdo con el alpha de Cronbach en esos estudios varía entre ..79 y ..89 (Diener, et al., 1985; Pavot, Diener, Colvin \& Sandvik, 1991). También, existe una amplia evidencia de la validez del SWLS en muestras españolas (Atienza et al., 2000 y Atienza, Balaguer, Corte-Real \& Fonseca, 2016). En el estudio actual, la fiabilidad de Cronbach fue $\alpha=.84$.

Cuestionario de bienestar. Este es un cuestionario desarrollado ad-hoc, para recoger información sobre el grado de satisfacción de los participantes en áreas específicas de 
su experiencia. Se compone de diecinueve ítems, de los cuales tres miden la satisfacción con los estudios (e.g. "Estoy satisfecho con mis resultados en los estudios"). Seis ítems miden la satisfacción con uno mismo (e.g. "Estoy orgulloso de ser como soy"). Siete ítems se refieren a la capacidad de resiliencia de los participantes (e.g. "Afronto de manera adecuada las situaciones estresantes") y tres ítems miden la satisfacción con el tiempo libre y de ocio (e.g. "En general me divierto bastante"). La consistencia interna de esta escala medida con el alpha de Cronbach fue de ..81. Las cuatro subescalas mostraron una fiabilidad de ..7. .85, ..78, ..75 respectivamente. El cuestionario se basa en otro anterior, utilizado con adultos (Pérez-Escoda \& Alegre, 2016), que mostró validez, fiabilidad y capacidad predictiva.

Competencia emocional. Se midió con un instrumento de inteligencia emocional percibida denominado Cuestionario de Desarrollo Emocional para educación secundaria (CDE-SEC; Pérez-Escoda, Bisquerra, Filella \& Soldevila, 2010). Es un cuestionario de auto-informe, desarrollado para población española, basado en el modelo teórico de educación emocional Bisquerra \& Pérez (2007). Su aplicación se estima adecuada entre los 12 y los 18 años. De acuerdo con este modelo, el cuestionario mide cinco dimensiones de la competencia emocional, además de ofrecer una puntuación global. Se compone de 35 ítems. Se utiliza una escala de tipo Likert con opciones de respuesta, que van desde 0 (completamente en desacuerdo con la declaración) a 10 (completamente de acuerdo). Estudios previos realizados con muestras de diversos niveles educativos indican que su índice de consistencia interna medida mediante el coeficiente alfa de Cronbach, oscila según los casos entre 0.79 y 0.82 (Pérez-Escoda, 2016). El presente estudio posee una fiabilidad de acuerdo con el alfa de Cronbach de .81 .

\section{Procedimiento y análisis de datos}

Se procedió, primero, a calcular los coeficientes de correlación de orden cero de Pearson entre las diferentes variables. Se comprobó de esta forma que las variables potencialmente predictoras de la satisfacción con la vida tenían relación con ésta. A continuación se realizó un análisis de regresión de la satisfacción con la vida en tres pasos. En el primer paso se introdujeron en la ecuación de regresión como variables independientes las satisfacciones experimentadas en contextos específicos (con los estudios, con uno mismo, con la capacidad de resiliencia, y con el uso del tiempo de ocio). El análisis de regresión de esta primera ecuación sirve para comprobar si las satisfacciones parciales predicen la satisfacción general con la vida.

En el segundo paso se introdujo la competencia emocional en la ecuación de regresión. De esta forma se comprobó la capacidad de predicción de esta variable, y el efecto que la introducción de esta variable en la ecuación de regresión puede tener sobre la capacidad de predicción de las satisfacciones parciales. Finalmente, en el tercer paso, se crearon cuatro variables moderadoras multiplicando la competencia emocional por cada una de las satisfacciones en contextos específicos. Se introdujeron estas nuevas variables en la ecuación de regresión, y se procedió a realizar un nuevo análisis de regresión. De esta forma se comprobó si la inteligencia emocional juega un papel mediador entre las satisfacciones en contextos específicos y la satisfacción con la vida. 


\section{Resultados}

\section{Datos descriptivos y correlaciones}

Los datos descriptivos se presentan en la tabla 1 y las correlaciones en la tabla 2. Se observa que los adolescentes en general se sienten bastante satisfechos en todas las áreas. Sin embargo manifiestan sentirse más satisfechos consigo mismos que en los otros tres contextos, e incluso más satisfechos consigo mismos que con su vida en general La segunda área de mayor satisfacción es la del uso de su tiempo de ocio. La menor satisfacción se da con los estudios.

En cuanto a las correlaciones, conviene destacar que las cuatro dimensiones de la escala de bienestar: satisfacción con uno mismo, con los estudios, con las actividades de ocio y la resiliencia correlacionan fuertemente tanto con la satisfacción con la vida como con la competencia emocional.

Tabla 1

Estadísticos Descriptivos

\begin{tabular}{lccc}
\hline & Media & Sd. & $\alpha$ \\
\hline Satisfacción con los estudios & 6.98 & 2.02 & .70 \\
Satisfacción con uno mismo & 8.18 & 1.54 & .85 \\
Satisfacción con los recursos de afrontamiento & 7.56 & 1.68 & .78 \\
Satisfacción con el tiempo de ocio & 7.87 & 1.96 & .75 \\
Competencia emocional & 6.21 & 1.10 & .81 \\
Satisfacción con la vida & 7.78 & 1.84 & .84 \\
\hline
\end{tabular}

Nota. $N=485$

Estos resultados permiten llevar a cabo un análisis de regresión ya que se ha comprobado que existe una relación estadística entre las diversas satisfacciones parciales y la competencia emocional con la satisfacción general con la vida.

Tabla 2

Correlaciones entre las variables del estudio

\begin{tabular}{lccccc}
\hline & $\begin{array}{c}\text { S. } \\
\text { estudios }\end{array}$ & $\begin{array}{c}\text { S. uno } \\
\text { mismo }\end{array}$ & $\begin{array}{c}\text { S. recursos } \\
\text { afrontamiento }\end{array}$ & $\begin{array}{c}\text { S. con } \\
\text { ocio }\end{array}$ & $\begin{array}{c}\text { C. } \\
\text { emocional }\end{array}$ \\
\hline Satisfacción uno mismo & $.634^{* *}$ & & & & \\
Satisfacción con recursos afrontamiento & $.628^{* *}$ & $.772^{* *}$ & & & \\
Satisfacción con ocio & $.547^{* *}$ & $.739^{* *}$ & $.726^{* *}$ & & \\
Competencia emocional & $.435^{* *}$ & $.539^{* *}$ & $.546^{* *}$ & $.497^{* *}$ & \\
Satisfacción con la vida & $.575^{* *}$ & $.688^{* *}$ & $.731^{* *}$ & $.616^{* *}$ & $.522^{* *}$ \\
\hline
\end{tabular}

Nota. $N=485 .{ }^{*} \mathrm{p}<.05,{ }^{* *} \mathrm{p}<.01$ 


\section{Análisis Predictivo}

El análisis de regresión confirmó que la satisfacción con los estudios, con uno mismo, y la satisfacción con los recursos de afrontamiento contribuyen a la predicción de la satisfacción con la vida. La satisfacción con el tiempo de ocio, en cambio, no aportó capacidad predictiva significativa (Tabla 3, Paso 1). Parece pues que los adolescentes distinguen entre ámbitos de la vida más importantes que otros. De esta forma, el ocio a pesar de ocupar una parte significativa de su tiempo, no influye en la valoración que los adolescentes hacen de su vida.

En el paso 2 de la Tabla 3 vemos también que cuando se incorpora la competencia emocional a la ecuación de regresión, ésta demuestra tener también poder de predicción sobre la satisfacción con la vida. Las satisfacciones con uno mismo, con la capacidad resiliencia, y con los estudios también conservan su capacidad de predicción. Estos resultados indican que en el caso de los adolescentes, tanto determinadas características inherentes a la persona (en este caso su inteligencia emocional), como otras que son consecuencia de las experiencias vividas por el adolescente (en nuestro estudio la satisfacción con los estudios, uno mismo, o la capacidad de resiliencia) realizan aportaciones independientes al sentimiento de satisfacción con la vida.

\section{Análisis de Moderación}

El análisis de regresión, añadiendo las variables predictivas y las variables moderadoras, mostró que la inteligencia emocional sólo modera la relación entre la resiliencia y la satisfacción con la vida, pero no la relación de las otras tres variables de bienestar: satisfacción con uno mismo, con los estudios, y con el ocio (Ver Tabla 3, Paso 3). Cuánto más emocionalmente inteligente es el adolescente, más influye la satisfacción que experimenta con su capacidad de resiliencia ante las adversidades en la satisfacción con la vida. Sin embargo eso no ocurre en los contextos de los estudios, del ocio, y de uno mismo.

Tabla 3

Análisis de regresión

\begin{tabular}{|c|c|c|c|c|c|c|}
\hline & \multirow{3}{*}{ Modelo } & \multicolumn{3}{|c|}{ Coeficientes } & \multirow{3}{*}{$\mathbf{t}$} & \multirow{3}{*}{ Sig. } \\
\hline & & \multicolumn{2}{|c|}{$\begin{array}{l}\text { Coeficientes No } \\
\text { Estandardizados }\end{array}$} & \multirow{2}{*}{$\begin{array}{c}\text { Coeficientes } \\
\text { Estandardizados } \\
\text { Beta }\end{array}$} & & \\
\hline & & B & Error Std. & & & \\
\hline \multirow{5}{*}{1} & (Constante) & 2.640 & 1.031 & & 2.561 & .011 \\
\hline & Satisfacción con los estudios & .132 & .042 & .124 & 3.122 & .002 \\
\hline & Satisfacción con uno mismo & .159 & .037 & .228 & 4.339 & .000 \\
\hline & Satisfacción con el tiempo de ocio & .075 & .051 & .069 & 1.465 & .144 \\
\hline & $\begin{array}{l}\text { Satisfacción con los recursos de } \\
\text { Afrontamiento }\end{array}$ & .232 & .028 & .427 & 8.312 & .000 \\
\hline
\end{tabular}




\begin{tabular}{|c|c|c|c|c|c|c|}
\hline \multirow{6}{*}{2} & (Constante) & .602 & 1.195 & & .504 & .615 \\
\hline & Satisfacción con los estudios & .121 & .042 & .114 & 2.899 & .004 \\
\hline & Satisfacción con uno mismo & .142 & .037 & .204 & 3.868 & .000 \\
\hline & Satisfacción con el tiempo de ocio & .060 & .051 & .055 & 1.177 & .240 \\
\hline & $\begin{array}{l}\text { Satisfacción con los recursos de } \\
\text { Afrontamiento }\end{array}$ & .216 & .028 & .398 & 7.706 & .000 \\
\hline & Competencia emocional & .692 & .211 & .118 & 3.283 & .001 \\
\hline \multirow{10}{*}{3} & (Constante) & -11.239 & 5.619 & & 2.000 & .046 \\
\hline & Satisfacción con los estudios & .613 & .250 & .579 & 2.450 & .015 \\
\hline & Satisfacción con uno mismo & -.079 & .213 & -.114 & -.373 & .709 \\
\hline & Satisfacción con el tiempo de ocio & -.100 & .289 & -.091 & -.345 & .730 \\
\hline & $\begin{array}{l}\text { Satisfacción con los recursos de } \\
\text { Afrontamiento }\end{array}$ & .523 & .154 & .961 & 3.394 & .001 \\
\hline & Competencia emocional & 2.760 & 1.012 & .471 & 2.727 & .007 \\
\hline & Satisfacción con los estudios x CE & -.080 & .041 & -.642 & 1.932 & .054 \\
\hline & Satisfacción con uno mismo x CE & .038 & .036 & .558 & 1.035 & .301 \\
\hline & $\begin{array}{l}\text { Satisfacción con los recursos de } \\
\text { Afrontamiento } \times \text { CE }\end{array}$ & -.053 & .026 & -.924 & 2.067 & .039 \\
\hline & Satisfacción con el tiempo de ocio x CE & .025 & .049 & .204 & .512 & .609 \\
\hline
\end{tabular}

Nota. $\mathrm{N}=485$. Variable dependiente: Satisfacción con la vida. $\mathrm{CE}=$ Competencia Emocional. Paso 1 ( $\mathrm{gl}=480$, $\mathrm{r}=.76)$. Paso $2(\mathrm{gl}=479, \mathrm{r}=.77)$. Paso $3(\mathrm{gl}=475, \mathrm{r}=.78)$.

\section{Discusión}

Este estudio plantea tres hipótesis. La primera hipótesis propone que la competencia emocional predice la satisfacción con la vida de los adolescentes. Una relación entre las competencias emocionales y la satisfacción con la vida, ya, había sido observada en estudios con adultos (Pérez-Escoda \& Alegre, 2016) y con estudiantes universitarios (Extremera \& Rey, 2016a). Sin embargo, casi no había evidencia de esta relación durante la adolescencia. Nuestro estudio confirma la relación entre competencia emocional y satisfacción con la vida durante esta etapa de la vida.

La satisfacción con la vida puede ser el resultado de haber disfrutado a lo largo de la vida, principalmente, de experiencias positivas y agradables, o puede deberse a una serie de habilidades emocionales de la persona que le permiten ver el lado positivo de cada experiencia vital (Diener, 1984). Las personas con mayor inteligencia emocional entienden las emociones, que motivan la conducta de las personas, lo que les permite sentirse menos víctimas de los comportamientos negativos desarrollados por otras personas y con mayores recursos para enfrentarlos. De esta forma, pueden derivar en una menor insatisfacción de las experiencias negativas y disfrutar más a fondo de las positivas.

Nuestra segunda hipótesis proponía la existencia, también, de una relación entre la satisfacción con la vida y cada uno de los dominios específicos de la satisfacción (satisfacciones con los estudios, con uno mismo, con los recursos de afrontamiento 
y con el uso del tiempo del ocio) en los jóvenes. Los resultados de este estudio indican que, efectivamente, todas las dimensiones de la satisfacción experimentada en contextos específicos se relacionan fuertemente con la satisfacción con la vida. Estudios anteriores habían mostrado ya una relación entre la satisfacción con la vida y la satisfacción con uno mismo (Rittenour \& Colaner, 2012), con la resiliencia (StackCutler, Parrila \& Torppa, 2015), con los estudios (Flynn \& Macleod, 2015) y con el tiempo de ocio (Ku, Fox \& Chen, 2016). Nuestro estudio, sin embargo es el primero que investiga la relativa aportación de cada una de estas áreas en la satisfacción con la vida de los adolescentes.

En este sentido, el análisis de regresión puso de manifiesto que sólo la satisfacción con uno mismo, con los estudios y con los recursos de afrontamiento concursan en la predicción de la satisfacción con la vida. La satisfacción con el tiempo de ocio no parece ser tan importante para los adolescentes. Este resultado parece indicar que los adolescentes son menos hedonistas de lo que, a menudo, se nos ha hecho creer y valoran más sus estudios, su propia persona y la capacidad de afrontar los retos que se les presentan que la diversión.

La tercera hipótesis proponía que la competencia emocional modera la relación entre satisfacción en cada uno de los dominios específicos y la satisfacción con la vida. Los resultados confirmaron esta hipótesis, para la satisfacción con los recursos de afrontamiento, pero no para la satisfacción en otros dominios específicos. En previos estudios se ha observado que la inteligencia emocional actúa como variable moderadora entre la satisfacción con la vida y las tendencias suicidas (Extremera \& Rey, 2016b), y entre el estrés psicológico y la satisfacción con la vida (Bhullar, Schutte \& Malouff, 2012). Los resultados de nuestro estudio aunque utilizan unas variables diferentes, son coherentes con estos estudios anteriores. Por su parte, en el trabajo de Limonero Tomás-Sábado, Fernández-Castro, Gómez-Romero \& Ardilla-Herrero (2012) se concluye, aunque con una muestra de universitarios, que la regulación emocional ejerce un efecto mediador en la satisfacción con la vida y en la forma de afrontar los acontecimientos estresantes. La capacidad de afrontar situaciones difíciles aumenta, cuando podemos entender las razones que mueven a las personas que las crean, las emociones envueltas en esas situaciones, y los efectos que nuestras acciones tendrán en las otras personas. Todo ello, hace que las personas con inteligencia emocional puedan sufrir menos en situaciones negativas y disfrutar más en las positivas, derivando, por tanto, de esas situaciones, mayor satisfacción que las personas con baja inteligencia emocional.

Los resultados de este estudio deben tomarse con precaución, debido a una serie de limitaciones. Primero, todos los datos provienen del auto informe de los adolescentes. Es sabido que los estudios de auto informe tienden a dilatar artificialmente las correlaciones entre las variables. También, todos los datos han sido obtenidos a través de cuestionarios y la utilización de un mismo método de obtención de datos tiende a incrementar las relaciones estadísticas. Adicionalmente, la muestra no es aleatoria, sino de conveniencia, por lo que la generalización a la población general debe hacerse con cautela. Por otra parte, se desconoce la situación socioeconómica de los participantes, una variable demográfica, que puede influir fuertemente en la satisfacción de vida. Sin embargo, sí se obtuvieron datos sobre el nivel educativo, que podrían servir 
como un indicador de la situación socioeconómica. No obstante, en nuestro estudio esta variable no afectó a los patrones de las relaciones entre las variables. En futuros estudios, se estima conveniente recoger información sobre otras variables potencialmente importantes, tales como la satisfacción con amigos íntimos o con amistades con las que se establece una relación romántica, relaciones que cobran importancia, sobre todo, en la adolescencia tardía. Al utilizar una muestra compuesta, en su mayor parte por chicos y chicas españoles, no es posible generalizar los resultados a otras culturas. Para investigar más a fondo las hipótesis, sería necesario desarrollar nuevos estudios, en los que también se midieran o se seleccionaran aquellas variables y poblaciones, antes mencionadas.

Destacamos, de los resultados obtenidos, ciertas tendencias, que es importante, que las tengamos en cuenta: Las experiencias positivas, en ámbitos concretos de la existencia, tales como los estudios, el desarrollo de capacidades de afrontamiento, la satisfacción con uno mismo y las capacidades emocionales, predicen la satisfacción con la vida. Además, el desarrollo de capacidades emocionales incrementa el efecto de las capacidades de afrontamiento en la satisfacción con la vida. Estas aportaciones empíricas son necesarias para conocer la realidad de los jóvenes (Cooper \& Sawaf, 1997; Weinsinger, 1997). Su contribución puede ser enriquecedora para el contexto educativo (Martínez-Antón et al., 2007) y para la motivación de los adolescentes en las tareas escolares (IFMB, 2008). Se recomienda, en consecuencia, intervenir en diversos niveles entre los cuales está el ámbito educativo (Bisquerra et al., 2015). Una forma de desarrollar la competencia emocional es mediante la intervención de programas de educación emocional en el curriculum escolar (Cejudo, López-Delgado \& Rubio, 2016; Pascual Ferris \& Cuadrado Bonilla, 2001; Sastre \& Moreno, 2002).

El aprendizaje del bienestar emocional y la auténtica felicidad es un reto, que tenemos pendiente en nuestra sociedad y en nuestras escuelas (Bisquerra, 2010).

\section{Referencias}

Atienza, F. L., Pons, D., Balaguer, I., \& García-Merita, M. (2000). Propiedades psicométricas de la escala de satisfacción con la vida en adolescentes. Psicothema, 12(2), 314-319.

Atienza, F. L., Balaguer, I., Corte-Real, N., \& Fonseca, A. M. (2016). Factorial invariance of the Satisfaction with Life Scale in adolescents from Spain and Portugal. Psicothema, 28(3), 353-358. doi: 10.7334/psicothema2016.1

Bermúdez, M. P., Álvarez, I. T., \& Sánchez, A. (2003). Análisis de la relación entre inteligencia emocional, estabilidad emocional y bienestar psicológico. Universitas Psychologica, 2(1), 27-32.

Bhullar, N., Schutte, N. S., \& Malouff, J. M. (2012). Trait Emotional Intelligence as a Moderator of the Relationship Between Psychological Distress and Satisfaction with Life. Individual Differences Research, 10, 19-26.

Bisquerra, R. (Coord.) (2010). La educación emocional en la práctica. Barcelona: Horsori-ICE. Bisquerra, R. (Coord.) (2011). ¿Cómo educar las emociones? La inteligencia emocional en la infancia y la adolescencia. Esplugues de Llobregat (Barcelona): Hospital Sant Joan de Déu. 
Bisquerra, R., \& Pérez, N. (2007). Las competencias emocionales. Educación XXI, 1. 61-82. Recuperado de http://dx.doi.org/10.5944/educxx1.1.10.297.

Bisquerra, R., Pérez-González, J. C., \& García-Navarro, E. (2015). Inteligencia emocional en la educación. Madrid: Síntesis.

Cava, M. J., \& Musitu, G. (1999). Percepción del profesor y estatus sociométrico en el grupo de iguales. Informació Psicològica, 71, 60-65.

Cazalla-Luna, N., Molero, D. (2016). Inteligencia emocional percibida, disposición al optimismo-pesimismo, satisfacción vital y personalidad de docentes en su formación inicial. Revista de Investigación Educativa, 34(1), 241-258. doi: 10.6018/rie.34.1.220701.

Cejudo, J., López-Delgado, M. L., \& Rubio M. J. (2016). Inteligencia emocional y resiliencia: su influencia en la satisfacción con la vida en estudiantes universitarios. Anuario de Psicología, 46, (2), 51-57. doi: 10.1016/j.anpsic.2016.07.001

Ciarrochi, J. V., Chan, A. C., \& Caputi, P. (2000). A critical evaluation of the emotional intelligence construct. Personality and Individual Differences, 28(3), 539-561. doi: 10.1016/S0191-8869(99)00119-1

Cooper, R. K., \& Sawaf, A. (1997). Estrategia emocional para ejecutivos. Barcelona: Martínez Roca.

Cho, E., \& Tay, L. (2015). Domain satisfaction as a mediator of the relationship between work-family spillover and subjective well-being: A longitudinal study. Journal of Business and Psychology, 1-13. doi: 10.1007/s10869-015-9423-8

Demir, M., \& Weitekamp, L. A. (2007). I am so happy cause today I found my friend: friendship and personality as predictors of happiness. Journal of Happiness Studies, 8, 181 - 211. doi: 10. 1007/s10902-006-9012-7

Diener, E. (1984). Subjective well-being. Psychological Bulletin, 95, 542-575.

Diener E., \& Gonzalez E. (2011). The validity of life satisfaction measures. Social Indicator NetworkNews, 108, 1-5

Diener, E., Emmons, R., Larsen, R. J. \& Griffin, S. (1985). The satisfaction with life scale. Journal of Personality Assessment,49, 71-75. Recuperado de http://dx.doi.org/10.1207/ s15327752jpa4901_13.

Diener, E., Suh, E. M., Lucas, R. E., \& Smith, H. L. (1999). Subjective well-being: Three decades of progress. Psychological Bulletin, 125, 276-302.

Extremera, N., Durán, A., \& Rey, L. (2005). La inteligencia emocional percibida y su influencia sobre la satisfacción vital, la felicidad subjetiva y el engagement en trabajadores de centros para personas con discapacidad intelectual. Ansiedad y Estrés, 11, 63-73.

Extremera, N., \& Rey, L. (2016a). Ability emotional intelligence and life satisfaction: Positive and negative affect as mediators. Personality $\mathcal{E}$ Individual Differences, 102, 98-101. doi: 10.1016/j.paid.2016.06.051.

Extremera, N., \& Rey, L. (2016b). Attenuating the Negative Impact of Unemployment: The Interactive Effects of Perceived Emotional Intelligence and Well-Being on Suicide Risk. PLoS ONE. 11, 1-13. doi: 10.1371/journal.pone.0163656.

Ferragut, M., \& Fierro, A. (2012). Inteligencia emocional, bienestar personal y rendimiento académico en preadolescentes. Revista Latinoamericana de Psicología, 44(3), 95-104.

Flynn, D. M., \& Macleod, S. (2015). Determinants of happiness in undergraduate university students. College Student Journal, 49, 452-460. 
Furr, R. M., \& Funder. D. (1998). A multimodal analysis of personal negativity. Journal of Personality and SocialPsychology, 74, 1580-1591. Recuperado de http://dx.doi. org/10.1037/0022-3514.74.6.1580.

Galindez, E., \& Casas, F. (2010). Adaptación y validación de la Students' Life Satisfaction Scale (SLSS) con adolescentes. Estudios de Psicología, 31(1), 79-87.

García Aguilar, N.; Viñals, M. \& Pérez-Escoda, N. (2016). Les competències emocionals i les dificultats quotidianes a l'adolescència. Comunicación presentada en las XII Jornadas de Educación Emocional; Barcelona: GROP-ICE de la Universidad de Barcelona.

Gonzáles- Barrón, R., Montoya, I.; Casullo, M., \& Bernabéu, J. (2002). Relación entre los estilos y estrategias de afrontamiento y bienestar psicológico en adolescentes. Psicothema, 14(2), 363-368.

Huebner, E. S. (1991a). Initial development of the Student's Life Satisfaction Scale. School Psychology International, 12, 231-240. doi: 10.1177/0143034391123010

Huebner, E. S. (1991b). Correlates of life satisfaction in children. School Psychology Quarterly, 6(2), 103-111. Recuperado de http://dx.doi.org/10.1037/h0088805

IFMB (Informe Fundación Marcelino Botín) (2008). Educación emocional y social. Análisis internacional. Santander: Fundación Marcelino Botín.

Javaloy, F., Páez, D., Cornejo, J. M., Besabe, N., Rodríguez, A., Valera, S., \& Espelt, E. (2007). Bienestar y felicidad de la juventud española. Madrid: Injuve.

Ku, P., Fox, K., \& Chen, L. (2016). Leisure-Time Physical Activity, Sedentary Behaviors and Subjective Well-Being in Older Adults: An Eight-Year Longitudinal Research. Social Indicators Research, 127, 1349-1361. DOI: 10.1007/s11205-015-1005-7.

Kuykendall, L., Tay, L., \& Ng, V. (2015). Leisure engagement and subjective well-being: A meta-analysis. Psychological bulletin, 141(2), 364. doi: 10.1037/a0038508

Lewinsohn, P. M., Redner, E. \& Seeley, J.R., (1991). The relationship between life satisfaction and psychosocialvariables: New perspectives. En F. Strack, M. Argyle \& N. Schwarz (Eds.), Subjective well- being: An interdisciplinary perspective (pp. 193212). New York: Pergamon.

Limonero, J. T.; Tomás-Sábado, J.; Fernández-Castro, J.; Gómez-Romero, Ma J. \& ArdillaHerrero, A. (2012) Estrategias de afrontamiento resilientes y regulación emocional: predictores de satisfacción con la vida. Behavioral Psychology / Psicología Conductual, 20(1), 183-196.

López, F., Etxebarria, I., Fuentes, M. J., \& Ortiz, M. J. (1999). Desarrollo afectivo y social. Madrid: Pirámide.

Lyubomirsky, S., King, L., \& Diener, E. (2005). The benefits of frequent positive affect: does happiness lead to success? Psychological bulletin, 131(6), 803. doi: 10.1037/00332909.131.6.803.

Martínez-Antón, M., Buelga, S., \& Cava, M. J. (2007). La satisfacción con la vida en la adolescencia y su relación con la autoestima y el ajuste escolar. Anuario de Psicología, 38(2), 293-303.

McCullough, G., Huebner, E. S., \& Laughlin, J. E. (2000). Life events, self-concept, and adolescents' positive subjective well-being. Psychology in the Schools, 37(3), 281-290. doi: 10.1002/(SICI)1520-6807(200005)37:3<281::AID-PITS8>3.0.CO;2-2. 
Montes-Berges, B., \& Augusto-Landa, J. M. (2014). Emotional intelligence and affective intensity as life satisfaction and psychological well-being predictors on nursing professionals. Journal of Professional Nursing, 30(1), 80-88.

Moreno, A. (2009). El desenvolupament durant l'adolescència. Barcelona: UOC.

Moreno, A., \& del Barrio, C. (2005). La experiencia adolescente. La búsqueda de un lugar en el mundo. Buenos Aires: Aique.

Moreno-Murcia, J. A., \& Silveira,Y. (2015). Perfiles motivacionales de estudiantes universitarios. Procesos de estudio y satisfacción con la vida. Revista Electrónica Interuniversitaria de Formación del Profesorado, 18(3), 169-181. doi: http://dx.doi. org/10.6018/reifop.18.3.200441

Mullis, R. L., \& Chapman, P. (2000). Age, gender, and self-esteem differences in adolescent coping styles. The Journal of Social Psychology, 140(4), 539-541. doi: 10.1080/00224540009600494

Pacheco, N. E., \& Fernández-Berrocal, P. (2004). El papel de la inteligencia emocional en el alumnado: evidencias empíricas. Revista electrónica de investigación educativa, 6(2), Documento electrónico. [Disponible en: http://redie.uabc.mx/vol6no2/contenidoextremera.html]

Páez, Fernández, Campos, Zubieta \& Casullo (2006). Apego Seguro, Vínculos Parentales, Clima Familiar e Inteligencia Emocional: socialización, regulación y bienestar. Ansiedad y estrés, 12, 329-341.

Pascual Ferris, V. \& Cuadrado Bonilla, M. (2001). Educación emocional. Programa de actividades para educación secundaria obligatoria. Barcelona: Wolters Kluwer.

Pavot, W., \& Diener, E. (2008). The satisfaction with life scale and the emerging construct of life satisfaction. The Journal of Positive Psychology, 3(2), 137-152. Recuperado de http://dx.doi.org/10.1080/17439760701756946

Pavot, W. G., Diener, E., Colvin, C. R., \& Sandvik, E. (1991). Further Validation of the Satisfaction with Life Scale: Evidence for the Cross-Method Convergence of WellBeing Measures. Journal of Personality Assess, 57, 149-161. http://dx.doi.org/10.1207/ s15327752jpa5701_17

Pérez-Escoda (2013). Variables predictivas de la satisfacción con la vida en estudiantes universitarios. Comunicación presentada al XVI Congreso Nacional / II Internacional Modelos de Investigación Educativa de AIDIPE, Alicante, 4-6 septiembre.

Pérez-Escoda, N. (2016). Cuestionarios del GROP para la evaluación de la competencia emocional (CDE). En J. L. Soler, L. Aparicio, O. Díaz, E. Escolano, y A. Rodríguez, Inteligencia emocional y bienestar II. Reflexiones, experiencias profesionales e investigaciones (pp. 690-705). Zaragoza: Ediciones Universidad San Jorge.

Pérez-Escoda, N., \& Alegre, A. (2014). Satisfacción con la vida: predictores y moderadores. En Barredo, B.; Bisquerra, R.; Blanco, A.; Garcia-Aguilar, N.; Giner, A.; PérezEscoda, N.; Tey, A. (Coords.). I Congreso Internacional de Educación Emocional. X Jornadas de Educación Emocional. Barcelona, Universitat de Barcelona (Institut de Ciencies de l'Educació), Documento electrónico. [Disponible en: http://hdl.handle. net/2445/58585]. Pp. 447-456.

Pérez Escoda, N., \& Alegre, A. (2016). Does Emotional Intelligence Moderate the Relationship between Satisfaction in Specific Domains and Life Satisfaction? International Journal of Psychology \& Psychological Therapy. 16(2), 131-140. 
Pérez-Escoda, N., \& Pellicer, I. (2009). Necesidad de desarrollo emocional en la adolescencia. I Jornades de Tutoria a l'ESO al segle XXI. Universitat de Barcelona, $<$ http://issuu.com/ eprat.ice.ub/docs/necesidad_de_desarrollo_emocional_en_la_adolescenc> [Consulta: 7 de febrero de 2016].

Pérez-Escoda, N., Álvarez, M., \& Bisquerra, R. (2008). CDE-SEC Cuestionario de desarrrollo emocional para Educación Secundaria. Barcelona: GROP. Universitat de Barcelona.

Pérez-Escoda, N., Bisquerra, R., Filella, G., \& Soldevila, A. (2010). Construcción del cuestionario de desarrollo emocional de adultos (QDE-A). REOP Revista Española de orientación psicopedagógica, 21(2), 367-379.

Pérez-González, J. C. (2011). Ability, stable self-perceptions, and competence are not the same thing: the case of 3 emotional intelligences. Paper presented at the 15th Biennial Inteligencia emocional en educacion Meeting of the International Society for the Study of the Individual Differences(IS-SID). Londres, UK, 25-28 July 2011.

Proctor, C., Tsukayama, E., Wood, A. M., Maltby, J., Eades, J. F.\& Linley, P. A. (2015) Gimnasia para las fortalezas: impacto de una intervención basada en las fortalezas del carácter sobre la satisfacción con la vida y el bienestar de los adolescentes. RET, Revista de Toxicomanías, 75, 10-23.

Rebollo-Catalán, A., Ruiz-Pinto, E. \& García-Pérez, R. (2017). Preferencias relacionales en la adolescencia según el género. Revista Electrónica de Investigación Educativa, 19(1), 58-72. Recuperado de http://redie.uabc.mx/redie/article/view/1022

Rittenour, Ch. \& Colaner, C. (2012). Finding Female Fulfillment: Intersecting RoleBased and Morality-Based Identities of Motherhood, Feminism, and Generativity as Predictors of Women's Self Satisfaction and Life Satisfaction. Sex Roles. 67, 351-362. doi: 10.1007/s11199-012-0186-7.

Roysamb, E., Nes, R. B., \& Vitterso, J. (2014). Well-being: Heritable and changeable. Stability of Happiness: Theories and Evidence on Whether Happiness Can Change, 9-36. doi: 10.1016/B978-0-12-411478-4.00002-3

Rutter, M. \& Rutter, M. (1992). Developing minds. Londres: Penguin.

Scheer, S., Unger, D., \& Brown, M. (1994). Adolescents becoming adults: attributes for adulthood. Comunicació presentada en la trobada bianual de la Society for Research on Adolescence, San Diego.

Salovey, P., \& Mayer, J. D. (1990). Emotional intelligence. Imagination, cognition and personality, 9(3), 185-211.

Sastre, G., \& Moreno, M. (2002). Resolución de conflictos y aprendizaje emocional. Barcelona: Gedisa.

Schuerger, J. M. (2003). Manual 16PF-APQ Cuestionario de Personalidad para Adolescentes 16PF. Madrid: TEA Ediciones, S.A.

Stack-Cutler, H. L., Parrila, R. K. \& Torppa,M. (2015). Using a Multidimensional Measure of Resilience to Explain Life Satisfaction and Academic Achievement of Adults With Reading Difficulties. Journal of Learning Disabilities, 48, 646-657.

Tay, L., \& Kuykendall, L. (2013). Promoting happiness: The malleability of individual and societal subjective wellbeing. International Journal of Psychology, 48, 159-176. doi: 10.1080/00207594.2013.779379 
Van Aken, M. A., \& Asendorpf, J. B. (1997). Support by parents, classmates, friends and siblings in preadolescence: Covariation and compensation across relationships. Journal of Social and Personal Relationships, 14(1), 79-93. doi: 10.1177/0265407597141004

Vázquez, C., Duque, A., \& Hervás, G. (2012). Escala de Satisfacción con la Vida (SWLS) en una muestra representativa de españoles adultos: Validación y datos normativos. Disponible en: http://pendientedemigracion.ucm.es/info/psisalud/ carmelo/PUBLICACIONES_pdf/2012-SWLS_Normative_data_copia.pdf

Veenhoven, R. (1994). El estudio de la satisfacción con la vida. Intervención Psicosocial, 3, 87-116.

Weisinger, H. (1997). Emocional intelligence at work: The untapped edge for success. San Francisco: Josey-Bass.

Ying, S., \& Fang-Biao, T. (2005). Correlations of school lifesatisfaction, self- esteem and coping style in middle schoolstudents. Chinese Mental Health Journal, 19, 741-744.

Fecha de recepción: 30 de octubre de 2016.

Fecha de revisión: 10 de mayo de 2017.

Fecha de aceptación: 10 de mayo de 2017. 
